\title{
AVALIAÇÃO DO DEBRIEFING NA SIMULAÇÃO CLINICANO ENSINO EM ENFERMAGEM
}

\author{
Maria Ercilia Chagas Rosa ${ }^{1}$ \\ Fernanda Maria Vieira Pereira-Ávila ${ }^{1}$ \\ Fernanda Garcia Bezerra Góes ${ }^{1}$ \\ Giovanna Martins de Salvo ${ }^{1}$ \\ Roberto Carlos Lyra da Silva ${ }^{2}$ \\ Verónica Rita Dias Coutinho ${ }^{3}$ \\ https://orcid.org/0000-0002-4130-7610 \\ https://orcid.org/0000-0003-1060-6754 \\ https://orcid.org/0000-0003-3894-3998 \\ https://orcid.org/0000-0001-8859-4621 \\ https://orcid.org/0000-0002-4327-6272 \\ https://orcid.org/0000-0001-8073-4562
}

Objetivo: avaliar o debriefing na prática simulada de graduandos de enfermagem. Método: estudo quantitativo em duas etapas: Etapa 1 - Realização de cenário de simulação clínica. Etapa 2 - Aplicação da Escala de Avaliação do Debriefing associado à Simulação (EaDaS). Todos os aspectos éticos foram atendidos. Resultados: participaram do estudo 36 (100,0\%) graduandos de enfermagem. Desses, 27 (75,0\%) relatou nunca ter participado de simulação. Na avaliação do debriefing associado à simulação, o escore geral obtido foi de 4,41 (DP=0,25), correspondendo ao percentual de 85,4\% de impacto positivo. A maioria dos graduandos 25 (69,4\%) confirmou que o debriefing auxiliou no desenvolvimento da capacidade para a tomada de decisões acertadas. Conclusão: a avaliação do debriefing foi positiva na simulação cínica, possibilitando melhorias no desempenho e aprendizado dos acadêmicos.

Descritores: Ensino; Simulação; Educação em enfermagem; Treinamento por simulação; Estudantes de enfermagem.

\section{NURSING EDUCATION: EVALUATING THE EFFECTIVENESS OF DEBRIEFING IN CLINICALSIMULATION}

Objective: To evaluate the debriefing in the simulated practice of nursing undergraduates. Method: Quantitative study in two steps: Step 1 - Realization of the clinical simulation scenario. Step 2 - Application of the Simulation Debriefing Assessment Scale (EaDaS). All ethical aspects were met. Results: 36 (100.0\%) nursing undergraduates participated in the study. Of these, 27 (75.0\%) reported never having participated in the simulation beforehand. In the evaluation of debriefing associated with the simulation, the overall score obtained was 4.41 (SD = 0.25), corresponding to a percentage of $85.4 \%$ of positive impact. Most undergraduates 25 (69.4\%) confirmed that debriefing assisted in developing the ability to make the right decisions. Conclusion: The evaluation of debriefing was positive in the clinical simulation, allowing improvements in the performance and learning of the students.

Descriptors: Teaching; Simulation; Nursing education; Simulation training; Nursing students.

\section{EVALUACIÓN DEBRIEFING EN LA SIMULACIÓN CLÍNICA EN LA EDUCACIÓN EN ENFERMERÍA}

Objetivo: Evaluarla información en la práctica simulada de estudiantes de pregrado en enfermería. Método: estudio cuantitativo en dos pasos: Paso l-Realización delescenario desimulaciónclínica. Paso 2:aplicación delaescala de evaluación de simulación deinformes(EaDaS). Todoslosaspectoséticosse cumplieron. Resultados: 36 (100.0\%) estudiantes universitarios de enfermería participaron en el estudio. De estos, 27 (75.0\%) informaron nunca haber participado en la simulación. En la evaluación de los informes asociados con la simulación, el puntaje general obtenido fue 4.41 (DE = 0.25), correspondiente a un porcentaje de $85.4 \%$ de impacto positivo. Lamayoría delosestudiantes universitarios 25(69.4\%)confirmaronquelareunión deinformaciónayudóa desarrollarlacapacidad detomarlas decisiones correctas. Conclusión: La evaluación del informe fue positiva en la simulación cínica, permitiendo mejoras en el rendimiento y el aprendizaje de los estudiantes. Descriptores: Enseñanza; Simulación; Educación en enfermería; Capacitación en simulación; Estudiantes de enfermería.

${ }^{3}$ Universidade Federal Fluminense. Rio das Ostras, RJ, Brasil.

2Universidade Federal do Estado do Rio de Janeiro. Rio de Janeiro, RJ, Brasil.

${ }^{3}$ Escola Superior de Enfermagem de Coimbra. Coimbra, Portugal.

Autor Correspondente: Maria Ercilia Chagas Rosa.E-mail: ercilia.chagas@gmail.com

Recebido: 21/11/2019 - Aceito: 06/08/2020 


\section{INTRODUÇÃo}

Os avanços tecnológicos e as atuais transformações sociais traduzem a necessidade da adoção de práticas inovadoras no ensino em saúde, incluindo estratégias que favoreçam o pensamento crítico do profissional a fim de promover uma abordagem clínica individualizada e segura ${ }^{()}$.

No contexto educacional, a reflexão acerca da segurança do paciente é gerada a partir de dois aspectos, um relacionado à garantia de que os alunos irão desenvolver habilidades essenciais para atender as necessidades do paciente de forma segura e outro referente ao método de ensino empregado, com a finalidade de contribuir para a formação acadêmica, evitando a exposição dos pacientes a riscos reais. Deste modo, torna-se imprescindivel que o aluno esteja capacitado para proceder de modo correto em situações mais complexas e limítrofes, identificadas no campo hospitalar ${ }^{(2,3)}$.

A elaboração de cenários realísticos tem sido empregada de maneira gradativa nos cursos de graduação em enfermagem e atualmente é considerado como um método diferenciado de ensino. Por meio desta técnica os alunos têm a possibilidade de obter conhecimentos de maneira prática, num ambiente seguro e facilitado por um professor ${ }^{(4)}$.

A simulação fornece, portanto, um cenário controlado de experiência prática para o processo de ensino aprendizagem, baseado no pensamento crítico e reflexivo individual e/ou coletivo. Logo, esta metodologia promove de maneira significativa o aprimoramento dos seus conhecimentos e habilidades. Por meio da simulação é possível dispor ao graduando a oportunidade de desenvolver segurança suficiente para realizar o manejo adequado do paciente no decorrer da assistência( ${ }^{(3-4)}$

Contudo, faz-se indispensável a realização de uma discussão reflexiva estruturada da experiência prática vivenciada na simulação clínica. Esse componente essencial e intencional da simulação, denominado debriefing, consiste em uma avaliação do aprendizado e do cuidado prestado ao paciente na sessão simulada, permitindo a associação do conteúdo teórico com a prática, a identificação das percepções e das atitudes dos participantes e o estabelecimento da sua autoconfiança ${ }^{(5)}$.

Recomenda-se que o debriefing ocorra durante ou após a simulação clínica viabilizando a análise da experiência vivenciada, por meio da utilização de instrumentos de pesquisa. Neste sentido, diversos autores têm construído e validado instrumentos para esta finalidade, como Student Satisfaction and Self-Confidence in Learning, Debriefing Experience Scale, Simulation Design Scale, Educational
Practices Questionaire e Escala de Avaliação do Debriefing associado à Simulação (EADaS)(5-8).

A EADaS construída e validada no idioma português tem o objetivo de avaliar o efeito do debriefing estruturado associado à prática simulada em estudantes de enfermagem. Os resultados obtidos por meio de sua utilização são tidos como indicadores essenciais para futuras pesquisas. ademais favorecem consideravelmente a validação científica dos resultados e auxiliam os docentes para estruturação de um roteiro de simulações ${ }^{(5,9)}$.

Considerando que a importância do uso de instrumentos válidos e confiáveis para avaliar o valor atribuído ao debriefing e que a EADaS apresenta boas propriedades psicométricas, o presente estudo teve como objetivo avaliar o impacto do debriefing na prática simulada de graduandos de enfermagem.

\section{MÉTODO}

\section{Tipo e Local de Estudo}

Trata-se de um estudo de natureza quantitativa, realizado no Laboratório de Ensino de Enfermagem de uma universidade pública localizada na baixada litorânea do estado do Rio de Janeiro no ano de 2018. O laboratório é equipado com manequins de baixa fidelidade, dispostos em camas hospitalares simulando um ambiente hospitalar. Apresenta divisórias entre os leitos, réguas de gases, mobiliário hospitalar, materiais para exame clínico, peças anatômicas sintéticas e monitores.

\section{Participantes do estudo}

A amostra do estudo foi composta por alunos do curso de enfermagem matriculados e regularmente inscritos no curso de enfermagem da universidade. Como critérios de inclusão foram considerados: discentes que haviam cursado a disciplina de Semiologia e Semiotécnica em Enfermagem; ter idade superior a 18 anos de idade.

Foi obtida uma lista nominal dos graduandos junto à coordenação de curso. No total, havia 100 alunos matriculados e regularmente ativos no terceiro, quarto e quinto ano. Desses, foram sorteados 40 graduandos, considerando-se a capacidade física do laboratório, os quais foram convidados por correio eletrônico. Após a confirmação individual de cada graduando, ocorreu uma reunião presencial para esclarecimentos acerca dos objetivos da pesquisa bem como o método a ser desenvolvido.

Mediante o aceite e a presença de 36 graduandos, os alunos foram divididos em quatro grupos de forma aleatória, com média de 9 alunos por grupo, e as simulações, com cenários iguais, foram agendadas em datas e horários dis- 
tintos para cada grupo e das atividades regulares do curso, e sem nenhuma interferência nas notas curriculares dos estudantes.

\section{Etapas do Estudo}

Primeira etapa: Realização de cenário de simulação clínica

Esta etapa compreendeu a execução de um cenário de simulação com avaliação clínica acerca dos sinais vitais de um paciente adulto. A cena foi estruturada utilizando simulador de baixa fidelidade posicionado no leito, estetoscópio, esfigmomanômetro, termômetro e equipamentos de proteção individual, para a representação de uma unidade clínica hospitalar. Foi elaborado um caso clínico que possibilitou a reprodução de situações objetivas com a participação de atores, a saber, duas monitoras acadêmicas e duas professoras, participantes da pesquisa, que se reuniram previamente para elaborar o caso e treinar suas ações e falas na cena, bem como organizaram o espaço para a simulação nos horários previstos. O tempo total de execução de cada cenário por grupo foi em média de 30 minutos.

\section{Segunda etapa: Aplicação do Debriefing associado à Simulação}

A segunda etapa compreendeu ao debriefing e à aplicação da EADaS e do questionário com informações individuais (sexo, data nascimento, período do curso e se já participou de simulação clínica anteriormente), com duração média de 30 minutos.

A EADaS é composta por 34 itens alocados em três domínios, valor psicossocial, valor cognitivo e valor afetivo, com opções de respostas em escala tipo Likert em cinco pontos, sendo: discordo completamente (1); discordo (2); nem concordo nem discordo (3); concordo (4); e concordo completamente (5)(5).

O domínio valor psicossocial contempla os itens 16, 17 , 19,21, 22, 23, 25, 26, 27, 28, 30, 32 e 33; o domínio valor afetivo os itens 2, 5, 9, 11, 14, 15, 18, 20, 24, 29, 3le 34; e, o domínio valor cognitivo os itens 1, 3, 4,6, 7, 8, 10, 12 e 13. Foram desenvolvidos 13 itens em sentido inverso, ou seja, aspectos tidos como negativos. Sendo assim, quanto maior o escore nos niveis de concordância "discordo completamente" e "discordo", melhor será o resultado no decorrer das análises.

\section{Análise de Dados}

Os dados foram analisados utilizando estatística descritiva, medidas de tendência central e de dispersão (média, moda, mediana, percentis, variância, desvio-padrão). O sorteio da amostra foi realizado por meio de números aleatórios gerados no IBM® SPSS® versão 22.0. O teste de Kolmogorov-Smirnov foi utilizado para verificar a normalidade das variáveis.
Para avaliação do debriefing, foi utilizado um escore médio de acordo com as respostas obtidas. O teste t de student foi utilizado para comparar o escore médio do debriefing associado à simulação e às variáveis sexo, idade e participação anterior em cenário de simulação. Para a comparação entre os grupos de alunos de acordo com o período de graduação, utilizou-se a análise de variância (ANOVA).

A análise dos escores ocorreu a partir da somatória dos itens de cada valor de domínio divido pelo total dos 34 itens que compõem a Escala de Aplicação do Debriefing associado à Simulação (EADaS). Para a avaliação dos resultados obtidos, foi considerado o valor 1,0 como mínimo, indicando um resultado negativo e para uma boa média o valor máximo foi de 5,0. A análise estatística foi feita por meio do software IBM® SPSS® versão 22.0.

\section{Aspectos Éticos}

O projeto foi submetido ao Comitê de Ética em Pesquisa da Faculdade de Medicina/Hospital Universitário Antônio Pedro da Universidade Federal Fluminense, número do Parecer: 1.602.948, CAAE: 55262616.0.0000.5243). Todos os aspectos éticos foram contemplados segundo a Resolução 466/2012 do Conselho Nacional de Saúde.

Os participantes foram esclarecidos quanto ao seu anonimato, sua participação voluntária, uso de pseudônimos, nenhuma interferência nas notas curriculares dos estudantes e a possibilidade de risco mínimo baseado em possivel desconforto em participar de uma atividade em grupo. Deste modo, foi solicitada a assinatura do Termo de Consentimento Livre e Esclarecido (TCLE) e as respostas obtidas foram tratadas de forma anônima e confidencial.

\section{RESULTADOS}

Participaram do estudo 36 (100,0\%) graduandos de enfermagem com predomínio do sexo feminino 33 (91,7\%), com idade maior ou igual a 21 anos 24 (66,7\%). Dos 40 graduandos que confirmaram presença, 04 (11,1\%) não compareceram. Do total de graduandos participantes, somente $09(25,0 \%)$ relataram ter participado de cenários de simulação (tabela 1).

Tabela 1 - Distribuição das variáveis individuais dos graduandos de enfermagem $(n=36)$. Rio das Ostras, RJ, 2018.

\begin{tabular}{|l|l|l|}
\hline Variáveis & N & $\%$ \\
\hline Sexo & & \\
\hline Feminino & 33 & 91,7 \\
\hline Masculino & 03 & 8,3
\end{tabular}




\begin{tabular}{lcc}
\hline Idade & & \\
\hline$\leq 21$ anos & 12 & 33.3 \\
\hline 21 anos & 15 & 66,7 \\
\hline Ano do curso & 9 & 41,7 \\
\hline 20 ano & 12 & 25,0 \\
\hline 3 @ ano & & 33,3 \\
\hline $4 \subseteq$ e 5 ano & \\
\hline Já participou de cenário & & \\
de simulação & 27 & 75,0 \\
\hline Não & 9 & 25,0 \\
\hline Sim & & \\
\hline
\end{tabular}

De acordo com as respostas dos graduandos de enfermagem para os itens da Escala, no que se refere ao item "aprender mais", 28 (77,8\%) consideraram o debriefing associado à prática simulada como uma estratégia relevante para o processo de ensino aprendizagem. Para o item "estruturar meu pensamento" 21 (58,3\%) concordaram totalmente (tabela 2).

Tabela 2 - Distribuição das frequências das respostas dos graduandos de enfermagem $(n=36)$ de acordo com os itens da Escala de Avaliação do Debriefing associado à Simulação (EADaS), Rio das Ostras, RJ, 2018.

\begin{tabular}{|c|c|c|c|c|c|}
\hline Itens & $\begin{array}{l}\text { I } \\
\mathrm{n}(\%)\end{array}$ & $\begin{array}{l}\text { II } \\
n(\%)\end{array}$ & $\begin{array}{l}\text { III } \\
n(\%)\end{array}$ & $\begin{array}{l}\text { IV } \\
n(\%)\end{array}$ & $\begin{array}{l}V \\
n(\%)\end{array}$ \\
\hline $\begin{array}{l}\text { 1-Estruturar o } \\
\text { meu pensamento }\end{array}$ & $\mathrm{O}(0,0)$ & $\mathrm{O}(0,0)$ & $\mathrm{O}(0,0)$ & $15(41,7)$ & $21(58,3)$ \\
\hline $\begin{array}{l}\text { 2-Me envergonhar } \\
\text { frente aos colegas } \\
\text { pelos meus erros }\end{array}$ & $14(38,9)$ & $15(41,7)$ & $5(13,9)$ & $2(5,6)$ & $\mathrm{O}(0,0)$ \\
\hline 3-Aprender mais & $\mathrm{O}(0,0)$ & $\mathrm{O}(0,0)$ & $\mathrm{O}(0,0)$ & $7(19,4)$ & $28(77,8)$ \\
\hline $\begin{array}{l}\text { 4-Me focar nos } \\
\text { aspectos impor- } \\
\text { tantes da atuação }\end{array}$ & $\mathrm{O}(0,0)$ & $1(2,8)$ & $3(8,3)$ & $11(30,6)$ & $20(55,6)$ \\
\hline $\begin{array}{l}\text { 5-Me deixar muito } \\
\text { ansioso/estres- } \\
\text { sado }\end{array}$ & $8(22,2)$ & $15(41,7)$ & $6(16,7)$ & $5(13,9)$ & $2(5,6)$ \\
\hline $\begin{array}{l}\text { 6-Refletir sobre as } \\
\text { minhas compe- } \\
\text { tências }\end{array}$ & $\mathrm{O}(0,0)$ & $\mathrm{O}(0,0)$ & $(0,0)$ & $8(22,2)$ & $28(77,8)$ \\
\hline $\begin{array}{l}\text { 7-Identificar } \\
\text { prioridades na } \\
\text { atuação }\end{array}$ & $\mathrm{O}(0,0)$ & $\mathrm{O}(0,0)$ & $3(8,3)$ & $10(27,8)$ & $23(63,9)$ \\
\hline $\begin{array}{l}\text { 8-Melhor identifi- } \\
\text { car os recursos a } \\
\text { utilizar na atuação }\end{array}$ & $\mathrm{O}(0,0)$ & $\mathrm{O}(0,0)$ & $3(8,3)$ & $14(38,9)$ & $19(52,8)$ \\
\hline $\begin{array}{l}\text { 9-Me humilhar } \\
\text { frente aos outros }\end{array}$ & $27(75,0)$ & $7(19,4)$ & $1(2,8)$ & $\mathrm{O}(0,0)$ & $1(2,8)$ \\
\hline
\end{tabular}

\begin{tabular}{|c|c|c|c|c|c|}
\hline $\begin{array}{l}\text { 10-Aprofundar } \\
\text { conhecimentos } \\
\text { específicos rela- } \\
\text { cionados com a } \\
\text { atuação }\end{array}$ & $\mathrm{O}(0,0)$ & $\mathrm{O}(0,0)$ & $l(2,8)$ & $18(50,0)$ & $17(47,2)$ \\
\hline $\begin{array}{l}\text { ll-Me deixar em } \\
\text { pânico só de } \\
\text { pensar em ter } \\
\text { de atuar de novo } \\
\text { numa situação } \\
\text { semelhante }\end{array}$ & $20(55,6)$ & $12(33,3)$ & $3(8,3)$ & $l(2,8)$ & $\mathrm{O}(0,0)$ \\
\hline $\begin{array}{l}\text { 12-Identificar } \\
\text { aspectos que devo } \\
\text { melhorar em atua- } \\
\text { ções futuras }\end{array}$ & $O(0,0)$ & $\mathrm{O}(0,0)$ & $l(2,8)$ & $6(16,7)$ & $29(80,6)$ \\
\hline $\begin{array}{l}\text { 13-Desenvolver } \\
\text { competências } \\
\text { para a tomada de } \\
\text { decisões acer- } \\
\text { tadas }\end{array}$ & $\mathrm{O}(0,0)$ & $\mathrm{O}(0,0)$ & $\mathrm{O}(0,0)$ & $10(27,8)$ & $25(69,4)$ \\
\hline $\begin{array}{l}\text { 14-Criar conflitos } \\
\text { no grupo }\end{array}$ & $32(88,9)$ & $3(8,3)$ & $l(2,8)$ & $O(0,0)$ & $\mathrm{O}(0,0)$ \\
\hline $\begin{array}{l}\text { 15-Não querer } \\
\text { participar em } \\
\text { mais nenhuma } \\
\text { simulação }\end{array}$ & $34(94,4)$ & $l(2,8)$ & $1(2,8)$ & $\mathrm{O}(0,0)$ & $\mathrm{O}(0,0)$ \\
\hline $\begin{array}{l}\text { 16-Aumentar a } \\
\text { minha autocon- } \\
\text { fiança }\end{array}$ & $1(2,8)$ & $\mathrm{O}(0,0)$ & $4(11,1)$ & $16(44,4)$ & $15(41,7)$ \\
\hline $\begin{array}{l}\text { 17-Desenvolver } \\
\text { competências de } \\
\text { liderança }\end{array}$ & $l(2,8)$ & $O(0,0)$ & $4(11,1)$ & $20(55,6)$ & $11(30,6)$ \\
\hline $\begin{array}{l}\text { 18-Eu me sentir } \\
\text { incompreendido }\end{array}$ & $21(58,3)$ & $11(30,6)$ & $3(8,3)$ & $l(2,8)$ & $\mathrm{O}(0,0)$ \\
\hline $\begin{array}{l}\text { 19-Aumentar o } \\
\text { potencial de tra- } \\
\text { balho em equipe }\end{array}$ & $O(0,0)$ & $\mathrm{O}(0,0)$ & $l(2,8)$ & $11(30,6)$ & $24(66,7)$ \\
\hline $\begin{array}{l}\text { 20-Eu me sentir } \\
\text { desrespeitado }\end{array}$ & $31(86,1)$ & $5(13,9)$ & $\mathrm{O}(0,0)$ & $\mathrm{O}(0,0)$ & $\mathrm{O}(0,0)$ \\
\hline $\begin{array}{l}21-E u \text { me sentir } \\
\text { realizado }\end{array}$ & $l(2,8)$ & $\mathrm{O}(0,0)$ & $13(36,1)$ & $20(55,6)$ & $2(5,6)$ \\
\hline $\begin{array}{l}\text { 22-Reforçar a mi- } \\
\text { nha iniciativa em } \\
\text { situações futuras }\end{array}$ & $\mathrm{O}(0,0)$ & $\mathrm{O}(0,0)$ & $l(2,8)$ & $14(38,9)$ & $21(58,3)$ \\
\hline $\begin{array}{l}\text { 23-Desenvolver a } \\
\text { relação de ajuda }\end{array}$ & $\mathrm{O}(0,0)$ & $\mathrm{O}(0,0)$ & $2(5,6)$ & $13(36,1)$ & $21(58,3)$ \\
\hline $\begin{array}{l}\text { 24-Eu sentir que } \\
\text { foi uma perda de } \\
\text { tempo }\end{array}$ & $35(97,2)$ & $l(2,8)$ & $\mathrm{O}(0,0)$ & $\mathrm{O}(0,0)$ & $\mathrm{O}(0,0)$ \\
\hline $\begin{array}{l}\text { 25-Reforçar a } \\
\text { minha autonomia } \\
\text { para atuar como } \\
\text { futuro enfermeiro }\end{array}$ & $O(0,0)$ & $\mathrm{O}(0,0)$ & $\mathrm{O}(0,0)$ & $13(36,1)$ & $23(63,9)$ \\
\hline $\begin{array}{l}\text { 26-Identificar difi- } \\
\text { culdades na minha } \\
\text { atuação }\end{array}$ & $0(0,0)$ & $0(0,0)$ & $\mathrm{O}(0,0)$ & $18(50,0)$ & $18(50,0)$ \\
\hline $\begin{array}{l}\text { 27-Promover a } \\
\text { autoconsciência } \\
\text { (conhecer as pró- } \\
\text { prias emoções) }\end{array}$ & $\mathrm{O}(0,0)$ & $\mathrm{O}(0,0)$ & $l(2,8)$ & $17(47,2)$ & $18(50,0)$ \\
\hline
\end{tabular}




\begin{tabular}{|c|c|c|c|c|c|}
\hline $\begin{array}{l}\text { 28-Eu me sentir } \\
\text { no centro do pro- } \\
\text { cesso formativo }\end{array}$ & $\mathrm{O}(0,0)$ & $2(5,6)$ & $9(25,0)$ & $21(58,3)$ & $4(11,1)$ \\
\hline $\begin{array}{l}\text { 29-Eu ter medo } \\
\text { de atuar no futuro } \\
\text { em situações } \\
\text { semelhantes }\end{array}$ & $15(41,7)$ & $10(27,8)$ & $6(16,7)$ & $5(13,9)$ & $O(0,0)$ \\
\hline $\begin{array}{l}\text { 30-Melhorar a } \\
\text { minha capacidade } \\
\text { de gerir emoções }\end{array}$ & $\mathrm{O}(0,0)$ & $\mathrm{O}(0,0)$ & $1(2,8)$ & $13(36,1)$ & $22(61,1)$ \\
\hline $\begin{array}{l}\text { 31-Bloquear o } \\
\text { meu raciocínio }\end{array}$ & $27(75,0)$ & $5(13,9)$ & $3(8,3)$ & $l(2,8)$ & $O(0,0)$ \\
\hline $\begin{array}{l}\text { 32-Eu sentir orgu- } \\
\text { lho por ser capaz } \\
\text { de executar mui- } \\
\text { tas intervenções } \\
\text { corretamente }\end{array}$ & $\mathrm{O}(0,0)$ & $3(8,3)$ & $8(22,2)$ & $16(44,4)$ & $9(25,0)$ \\
\hline $\begin{array}{l}\text { 33-Eu sentir } \\
\text { que o professor } \\
\text { tem interesse } \\
\text { genuino no meu } \\
\text { desenvolvimento } \\
\text { profissional }\end{array}$ & $l(2,8)$ & $\mathrm{O}(0,0)$ & $1(2,8)$ & $19(52,8)$ & $15(41,7)$ \\
\hline $\begin{array}{l}\text { 34-Baralhar as } \\
\text { minhas ideias } \\
\text { a respeito da } \\
\text { atuação }\end{array}$ & $11(30,6)$ & $13(36,1)$ & $4(11,1)$ & $6(16,7)$ & $2(5,6)$ \\
\hline
\end{tabular}

*Nota: I - Discordo totalmente; II - Discordo; III - Nem concordo nem discordo; IV - Concordo; V - Concordo totalmente.

Em relação à questão acerca de "refletir sobre minhas competências", 28 (77,8\%) concordaram totalmente, afirmando que este método promove a reflexão acerca de suas habilidades. No que tange ao item "identificar aspectos que devo melhorar em atuações futuras", 29 (80,6\%) concordaram totalmente que o debriefing estruturado associado à prática simulada auxilia na identificação de aspectos que devem ser aprimorados para uma melhor atuação em futuras circunstâncias.

Para os itens com sentidos inversos, "Criar conflitos no grupo" 32 (88,9\%) e "não ter interesse em participar de futuras simulações" 34 (94,4\%), alunos discordaram totalmente dessas afirmações.

O item referente à autoconfiança apresentou resultado positivo, demonstrando que 31 (86,1\%) dos alunos concordaram totalmente ou concordaram que debriefing estruturado contribui para o desenvolvimento da autoconfiança.

Do total de alunos, 24 (66,7\%) atestaram de maneira positiva que o debriefing aumenta o potencial de trabalho em equipe. Com relação ao item "eu sentir que foi uma perda de tempo" 35 (97,2\%) discordaram totalmente que essa prática pode promover este sentimento.
Ainda, 23 (63,9\%) graduandos concordaram totalmente no tópico "reforçar a minha autonomia para atuar como futuro enfermeiro". Logo, 18 (50,0\%) participantes concordaram totalmente que houve contribuição para identificar as dificuldades na atuação.

Quanto ao item "Eu sentir orgulho por ser capaz de executar muitas intervenções corretamente", 16 (44,4\%) graduandos julgaram a simulação como um meio de executar os procedimentos inúmeras vezes até realizá-los de maneira correta, ocasionando desta forma na satisfação dos mesmos em dispor de competência considerável em poder atuar com convicção em situações reais.

$\mathrm{Na}$ avaliação da Escala o escore geral obtido foi de 4,41 (DP=0,25), correspondendo o percentual de $85,4 \%$ de impacto positivo do debriefing associado à simulação nos graduandos de enfermagem. Na comparação entre grupos, não houve diferença estatisticamente significativa (IC $95 \%)$ entre o sexo $(p=0,096)$, idade $(p=0,257)$, período do curso $(p=0,183)$ e participação anterior em cenário de simulação $(p=0,356)$ (tabela 3).

Tabela 3 - Comparação dos escores do impacto do debriefing segundo variáveis individuais. Rio das Ostras, RJ, 2018

\begin{tabular}{|c|c|c|}
\hline Variáveis & Escore (\%) & $\begin{array}{l}\text { Teste (valor de p IC } \\
95 \% \text { ) }\end{array}$ \\
\hline \multicolumn{3}{|l|}{ Sexo } \\
\hline Feminino & $4,40(85,2)$ & $t=-1,71(0,096)$ \\
\hline Masculino & $4,65(91,4)$ & \\
\hline \multicolumn{3}{|l|}{ Idade } \\
\hline$\leq 21$ anos & $4,50(87,6)$ & $t=-1,15(0,257)$ \\
\hline$>21$ anos & $4,39(84,9)$ & \\
\hline \multicolumn{3}{|c|}{ Periodo do curso } \\
\hline 4 e 5 & $4,47(86,9)$ & $t=1,79(0,183)$ \\
\hline 6 e 7 & $4,30(82,5)$ & \\
\hline 8 ou mais & $4,47(86,5)$ & \\
\hline \multicolumn{3}{|c|}{$\begin{array}{l}\text { Já participou de cenário } \\
\text { de simulação }\end{array}$} \\
\hline Não & $4,40(85,1)$ & $t=-0,93(0,356)$ \\
\hline Sim & $4,49(87,4)$ & \\
\hline
\end{tabular}

Fonte: Coleta de dados da pesquisa.

Foram obtidos os escores segundo os domínios cognitivo 4,61 (DP=0,37), afetivo 4,09 (DP=0,29) e psicossocial $4,29$ ( $D P=0,36)$. Na comparação dos escores do domínio psicossocial entre os graduandos e participação em simulação houve diferença estatisticamente significante ( $t=-$ $2,11 ; p=0,042)$, ou seja, os graduandos que relataram já ter 
participado de simulação apresentaram um escore maior $4,50(\mathrm{DP}=0,04)$ para este domínio quando comparados aos que nunca haviam participado 4,22 (DP=0,31).

\section{DISCUSSÃO}

Dentre as características da amostra estudada, observa-se que se trata de uma população jovem. Destaca-se que a inserção dos jovens com idade entre 18 a 24 anos nas universidades tem aumentado razoavelmente nos últimos anos devido à implementação de políticas de expansão e democratização do acesso, esse movimento resultou no aumento do quantitativo da oferta de novos cursos de graduação nas instituições públicas e privadas, possibilitando que mais jovens ingressem no ensino superior em diversas regiões do Brasil(10-12), o que corrobora com o perfil dos graduandos do cenário da pesquisa, localizado no interior do estado do Rio de Janeiro.

A sociedade progressivamente tem exigido dos profissionais em saúde, melhorias referentes à qualificação e segurança na assistência em prol do bem-estar da população. Logo, a simulação clínica possibilita a execução e treinamento de procedimentos terapêuticos de forma segura, em prol do cuidado ético e responsável ao paciente, família e comunidade ${ }^{13-14}$. Ademais, outros autores confirmam que a simulação resulta em um efeito benéfico para o controle do nível de ansiedade comum entre estudantes ${ }^{(15,3)}$

Estudos apontam ainda que a simulação na graduação é eficaz quando empregada como método educacional, resultando no aumento da autoconfiança e estimulação para a construção do pensamento crítico e clínico do aluno, bem como no compartilhamento de conhecimentos e experiências, contribuindo de maneira considerável para sua formação acadêmica (4,16,17). Sendo assim, considera-se fundamental a utilização de estratégias educativas dessa natureza na aprendizagem, fundamentada em casos reais estimulando, desse modo, o crescimento e a autoconfiança dos graduandos, além da segurança dos pacientes.

Por outro lado, é essencial a existência de instrumentos que permitam a avaliação de forma objetiva dos resultados dessa metodologia de ensino. Nessa linha, o debriefing é uma estratégia que permite aos participantes explorarem, analisarem e sintetizarem pensamentos, processos, estados emocionais e outros aspectos numa experiência simulada, visando a melhorar o seu desempenho em situações reais $^{(18)}$. Assim, os achados da presente pesquisa apontaram para o impacto positivo do debriefing associado à prática simulada de graduandos de enfermagem com a utilização de uma escala válida e confiável, a EADaS.
Por meio desse instrumento, evidenciou-se que os graduandos concordaram que o debriefing estruturado associado à prática simulada auxilia de modo considerável no aprendizado referente à prática profissional e ao trabalho em equipe. Corroborando com esses resultados, estudo desenvolvido na Escola de Enfermagem de Coimbra, Portugal, verificou que os alunos também consideraram o debriefing estruturado como um método interativo que permite consolidar e sistematizar seu conhecimento, refletir individual e coletivamente sobre as atividades e estruturar suas ideias ${ }^{(18)} \mathrm{o}$ que reforça a importância dessa prática e do uso de instrumentos válidos e confiáveis para avaliá-la de forma estruturada.

Nesse mesmo cenário, outra investigação constatou que o debriefing estruturado em associação com simulação tem maior impacto para os alunos em comparação com o feedback tradicional, com evidentes contribuições para a aprendizagem, pois o grupo experimental, que recebeu o debriefing estruturado, apresentou maiores médias em todos os dimensões e na EADaS global, bem como diferenças estatisticamente significativas do grupo controle, com feedback tradicional(19)

O debriefing associado à simulação permitiu ainda que, os alunos se sentissem mais à vontade para fazer perguntas e estabelecer uma relação mais próxima com seus colegas, facilitando comunicação e melhorando suas habilida$\operatorname{des}^{(17)}$, o que corrobora com os achados atuais. Além disso, foi possivel observar, por meio da EADaS, que os graduandos da presente pesquisa avaliaram o debriefing como uma possibilidade de aprender mais, refletir sobre suas competências para a tomada de decisões acertadas e identificar prioridades para sua futura atuação como profissional.

Quanto à possibilidade de participar de uma nova simulação, foi observado o desejo dos alunos em integrar-se novamente nessa experiência metodológica, além do mais afirmaram que o debriefing não consiste numa perda de tempo. Vale ressaltar que a realização dos cenários propicia a oportunidade de relacionar as habilidades clínicas, conhecimento científico, avaliação terapêutica de enfermagem, comunicação interdisciplinar e o pensamento crítico em tempo real, assim como o desenvolvimento da autoconfiança no cuidado ao paciente ${ }^{(14)}$.

Por intermédio da simulação é possivel dispor de um ambiente realístico, favorecendo para o desenvolvimento de competências psicossociais, cognitivas e emocionais. Desta forma, auxilia na estruturação do pensamento crítico, no autocontrole, aprimoramento dos conhecimentos obtidos com a teoria e como trabalhar em equipe ${ }^{(20,3)}$, que pode ter seu efeito avaliado por meio da EADaS, conforme confirmado no achados do estudo. 
Cada domínio da EADaS, utilizada nesta pesquisa, foi submetida a uma análise com a finalidade de avaliar os efeitos da aprendizagem ativa ocasionados por essa abordagem educacional associada à simulação clínica. O domínio cognitivo está relacionado ao desenvolvimento de competências intelectuais. Em contrapartida, o afetivo refere-se ao modo de enfrentar as situações emocionalmente, como euforia, medo, atitude, tristeza, motivação e valor. No que se refere às questões psicológicas e sociais na simulação, estão relacionadas ao domínio psicossocial da EADaS ${ }^{(18,19)}$. Pesquisa aponta que quando associado à prática simulada, o debriefing tem um impacto muito positivo no desenvolvimento de habilidades dos estudantes de enfermagem nas dimensões psicossocial, cognitiva e afetiva(19).

No decorrer das análises constatou-se que a menor parte do grupo já havia tido experiências anteriores com a simulação, ainda assim, no domínio psicossocial que este grupo apresentou um escore maior comparado aos demais graduandos que nunca tiveram experiências com a simulação, comprovando que a participação prévia em simulações interfere no efeito do debriefing associado à prática simulada.

Estudo recente, realizado na Universidade Federal do Paraná, com 35 estudantes do curso de enfermagem, utilizou outra escala de avaliação do debriefing associado à simulação, nas dimensões psicossocial, cognitiva e afetiva. A média geral dos níveis de concordância da escala foi de 3,90, visto que o domínio cognitivo apresentou um escore maior, seguido pelo fator psicossocial. Os resultados dessa pesquisa reforçaram que a fase do debriefing promove um processo de aprendizagem reflexiva com a oportunidade de desenvolver habilidades distintas ${ }^{(21)}$, o que também corrobora com os achados referentes ao domínio cognitivo considerando seu maior escore entre os participantes do estudo.

\section{Limitações do estudo}

Como limitação do estudo destaca-se a capacidade física do laboratório, impossibilitando que todos os graduandos participassem da simulação no mesmo dia, e o uso de simulador de baixa fidelidade. Para resultados mais precisos, salienta-se a realização de futuras pesquisas em um laboratório com espaço físico apropriado, de acordo com a quantidade de participantes.

\section{Contribuições para a prática}

Os resultados do estudo corroboram para importância da utilização da simulação clínica no processo de ensino aprendizagem no ensino da enfermagem. A partir do momento que esse processo se coloca em prática, formam-se enfermeiros com maior capacidade de tomadas de deci- sões gerenciais e assistenciais de qualidade, com habilidade aprimorada, uma vez que ele é um facilitador da prática e permite o desenvolvimento do pensamento crítico e construtivo.

\section{CONCLUSÃO}

A avaliação do debriefing foi positiva na simulação clínica. A análise dos domínios psicossocial, afetivo e cognitivo possibilitou evidenciar os efeitos benéficos proporcionados pelo debriefing na simulação clínica, dentre eles, o aprimoramento das competências, desenvolvimento da autoconfiança e do trabalho em equipe.

A Escala de Avaliação do Debriefing associado à Simulação (EADaS) aplicada no presente estudo possibilitou identificar valores notáveis quanto aos escores das variáveis e dos domínios. No decorrer da avaliação constatou-se uma média maior referente ao fator cognitivo em relação aos demais domínios, contudo, dentre os escores do domínio psicossocial, evidenciou uma diferença considerável no que tange a habilidade dos graduandos que já haviam participado de simulação clínica durante o seu percurso acadêmico.

Ressalta-se que o desenvolvimento de competência clínica foi confirmado a partir dos resultados apontados pela EADaS. Acerca dos aspectos psicossociais, a análise revelou que o debriefing possibilita que o aluno desenvolva capacidades para tomada de decisões, comunicação, consolidação da autonomia para atuar futuramente, além de promover a autoconsciência e, consequentemente, melhores práticas no contexto clínico e mais ganhos na assistência em saúde.

\section{Contribuição dos autores:}

Maria Ercília Chagas Rosa - desenho do estudo, coleta de dados, análise e interpretação dos dados, redação do artigo, revisão crítica, revisão final. Fernanda Maria Vieira Pereira-Ávila - concepção, desenho do estudo, coleta de dados, análise e interpretação dos dados, redação do artigo, revisão crítica, revisão final. Fernanda Garcia Bezerra Góes - concepção, desenho do estudo, coleta de dados, análise e interpretação dos dados, redação do artigo, revisão crítica, revisão final. Giovanna Martins de Salvo - coleta de dados, análise e interpretação dos dados. Roberto Carlos Lyra da Silva - coleta de dados, análise e interpretação dos dados, redação do artigo. Verónica Rita Dias Coutinho - análise e interpretação dos dados, redação do artigo.

\section{Agradecimento:}

Ao Conselho Nacional de Desenvolvimento Científico e Tecnológico - CNPq pela concessão da bolsa de iniciação científica. 


\section{REFERÊNCIAS}

1. Domingues NA, Tibes CM, Dias JD, Westin UM, Zem-Mascarenhas SH, Fonseca LMM. Simulação virtual por computador no ensino de enfermagem: relato de experiência. Rev Enferm UFPI. 2017 [citado 25 Jan 2019]; 6(4): 70-74. Disponível em: http://www.ojs.ufpi.br/index.php/reufpi/article/ view/6174/pdf

2. Oliveira SN, Massaroli A, Martini JG, Rodrigues J. From theory to practice, operating the clinical simulation in Nursing teaching. Rev Bras Enferm [Internet]. 2018 [citado 30 Out 2018];71 (Suppl 4): 1791-8. Disponivel em: https:// www.scielo.br/pdf/reben/v7ls4/pt_0034-7167-reben-71-s4-1791.pdf.

3. Mesquita HCT, Santana BS, Magro MCS. Efeito da simulação realística combinada à teoria na autoconfiança e satisfação de profissionais de enfermagem. Esc Anna Nery Rev Enferm. 2019 [citado 25 Jan 2019]: 23(10). Disponivel em: http://www.scielo.br/pdf/ean/v23nl/pt_1414-8145-ean23-01-e20180270.pdf

4. Oliveira SN, Prado ML, Kempfer SS. Utilização da simulação no ensino da enfermagem: revisão integrativa. REME. 2014 [citado 10 Dez 2018]; 18(2): 487-495. Disponivel em: http://www.reme.org.br/artigo/detalhes/941

5. Coutinho VRD, Martins JCA, Pereira MFCR. Construção e Validação da Escala de Avaliação do Debriefing associado à Simulação (EADaS). Rev Enf Ref [Internet]. 2014 [citado 30 Set 2018]; 4(2): 41-50. Disponivel em: http:// www.scielo.mec.pt/pdf/ref/vserIVn2/serIVn2a05.pdf

6. Almeida RGS, Mazzo A, Martins JCA, Coutinho VRD, Jorge BM, Mendes IAC. Validation to Portuguese of the Debriefing Experience Scale. Rev Bras Enferm [Internet]. 2016 [citado 20 Abr 2019]; 69(4): 658-64. Disponivel em: http://www.scielo.br/pdf/reben/v69n4/0034-7167-reben-69-04-0705.pdf

7. Unver V, Basakb T, Wattsc P, Gaiosoc V, Mossc J, Tastand S, et al. The reliability and validity of three questionnaires: The Student Satisfaction and Self-Confidence in Learning Scale, Simulation Design Scale, and Educational Practices Questionnaire. Contemp Nurse [Internet]. 2017 [citado 20 Jan 2019]: 53(1): 60-74. Disponivel em: https://toad.edam.com.tr/sites/ default/files/pdf/the-student-satisfaction-and-self-confidence-in-learning-scale-toad.pdf

8. Bergamasco EC, Murakami BM, da Cruz DALM. Uso da Escala de Satisfação dos Estudantes e Autoconfiança com a Aprendizagem (ESEAA) e da Escala do Design da Simulação (EDS) no ensino de enfermagem: relato de experiência. Sci Med (Porto Alegre, Online). [Internet]. 2018 [citado 26 Fev 2019]; 28(3): ID31036. Disponivel em: http: //revistaseletronicas.pucrs.br/ ojs/index.php/scientiamedica/article/viewFile/31036/17103

9. Mayara MMP, Fabiola LR, Luciana MM, Lara MMB, Manuela CM, Fabiana CVF, Geisa S. Avaliação do processo ensino-aprendizagem no ambiente de simulação realística na graduação em enfermagem. Enferm foco [Internet]. 2019 [citado 14 Jul 2019]; 10 (6): 118-124. Disponivel em: http://revista. cofen.gov.br/index.php/enfermagem/article/view/2782/660

10. Zluhan MR, Raitz TR. A educação em direitos humanos para amenizar os conflitos no cotidiano das escolas. RBEP. 2014 [citado 20 Jan 2019]; 95(239): 31-54. Disponivel em: http://www.scielo.br/scielo.php?pid=S217666812014000100003Escript=sci_abstract\&tlng=t

11. Trevisol JV, Nierotka RL. Os jovens das camadas populares na universi- dade pública: acesso e permanência. Rev Katál [Internet]. 2016 [citado 30 Jan 2019]; 19(1): 22-32. Disponivel em: http://www.scielo.br/scielo.php?pi$d=S 1414-49802016000100022 \varepsilon$ script=sci_abstract\&tlng=pt

12. Leticia KM, Rosa MR, Raphael KS, Solange FRC, Mateus SL. Expansão dos cursos de graduação em enfermagem no Brasil entre 2004 e 2017. Enferm foco [Internet]. 2019 [citado 14 Jul 2019]; 10 (6): 63-69. Disponivel em: http://revista.cofen.gov.br/index.php/enfermagem/article/view/2369/652

13. Quirós SM, Vargas MAO. Simulação clínica: uma estratégia que articula práticas de ensino e pesquisa em Enfermagem. Texto \& Contexto Enferm [Internet]. 2014 [citado 30 Set 2018]; 23(4): 813-814. Disponivel em: http:// www.scielo.br/pdf/tce/v23n4/pt_0104-0707-tce-23-0400815.pdf

14. Valadares AFM, Magro MCS. Opinião dos estudantes de enfermagem sobre a simulação realística e o estágio curricular em cenário hospitalar. Acta Paul Enferm (Online) [Internet]. 2014 [citado 30 Set 2018]; 27(2): 138-143. Disponivel em: http://www.scielo.br/scielo.php?pid=S010321002014000200009\&script=sci_abstract\&t>lng =ES

15. Teixeira CRS, Pereira MCA, Kusumota L, Gaioso VP, Mello CL, Carvalho EC Evaluation of nursing students about learning with clinical simulation. Rev Bras Enferm [Internet]. 2015 [citado 30 Jan 2019]; 68(2): 311-9. Disponivel em: <http://www.scielo.br/pdf/reben/v68n2/0034-7167-reben-68-02-031l.pdf

16. Kimhi E, Reishtein JL, Cohen M, Friger M, Hurvitz N, Avraham R. Impact of Simulation and Clinical Experience on Self-efficacy in Nursing Students: Intervention Study. Nurse Educ [Internet]. 2016 [citado em 10 Fev 2019]; 41(1):El-4. Disponivel em: https://insights.ovid.com/pubmed?pmid $=26218009$

17. Cogo ALP, Lopes EFS, Perdomini FRI, Flores GE, Santos MRR. Construção e desenvolvimento de cenários de simulação realistica sobre a administração segura de medicamentos. Rev Gaúch Enferm [Internet]. 2019 [citado em 25 Jan 2019]; 40(esp):e 20180175. Disponivel em: http://www.scielo. br/scielo.php?script=sci_arttext\&pid=S1983-14472019000200801\&lng=en

18. Coutinho V, Martins JA, Pereira F. Structured debriefing in Nursing simulation: students' perceptions. J Nurs Educ Pract [Internet]. 2016 [citado em 25 Jan 2019]; 6(9):127-134. Disponivel em: http://www.sciedu.ca/journal/index.php/jnep/article/view/8878/5824.

19. Coutinho V. Martins JA. Pereira F. Student's opinion about feedback VS debriefing: a randomized controlled trial. IJND [Internet]. 2017 [citado em 25 Jan 2019]; 7:63-68. Disponivel em: https://www.researchgate.net/ publication/320430492_Student's_opinion_about_feedback_Vs_structured_debriefing_a_randomized_controlled_trial.

20. Çelik Y, Ceylantekin Y, Kiliç I. The evaluation of simulation market in nursing education and the determination of learning style of students. Int $J$ Health Sci (Oassim) [Internet]. 2017 [citado 25 Jan 2019]; 11(1): 16. Disponivel em https://www.ncbi.nlm.nih.gov/pmc/articles/PMC5327665/

21. Major CB, Mantovani MF, Félix JVC, Boostel R, Silva ATM, Caravaca-Morera JA. Avaliação do debriefing na simulação clínica em enfermagem: um estudo transversal. Rev Bras Enferm [Internet]. 2019 [citado 30 Abr 2019]; 72(3): 825-31. Disponivelem: http://www.scielo.br/scielo.php?script=sci_ arttext\&pid=S003471672019000300788\&lng=en\&nrm=iso\&tlng=pt 